\section{Caracterização de demandas judiciais de fornecimento de medicamentos "essenciais" no Estado do Rio de Janeiro, Brasil}

\author{
Characterization of lawsuits for the supply of \\ "essential" medicines in the State of \\ Rio de Janeiro, Brazil
}

\author{
Vera Lúcia Edais Pepe 1 \\ Miriam Ventura 1 \\ João Maurício Brambati Sant'ana 1 \\ Tatiana Aragão Figueiredo 1 \\ Vanessa dos Reis de Souza 1 \\ Luciana Simas 1 \\ Claudia Garcia Serpa Osorio-de-Castro ${ }^{1}$
}

\section{Introdução}

\footnotetext{
${ }^{1}$ Escola Nacional de Saúde Pública Sergio Arouca,

Fundação Oswaldo Cruz, Rio

de Janeiro, Brasil.

Correspondência

V. L. E. Pepe

Departamento de

Administração e

Planejamento em Saúde,

Escola Nacional de Saúde

Pública Sergio Arouca,

Fundação Oswaldo Cruz.

Rua Leopoldo Bulhões 1480

sala 711 , Rio de Janeiro, $R J$

21041-210, Brasil.

verapepe@ensp.fiocruz.br
}

\begin{abstract}
Recognition of the right to health raises two practical issues: the government's ethical and legal duty to ensure comprehensive health care and citizens' recourse to legal action to guarantee this right. This study focused on lawsuits to demand "essential" medicines, filed at the State Court of Appeals in Rio de Janeiro, Brazil, in 2006. One hundred and eighty-five suits were examined, and the claims were granted in all but three cases. Median times between filing the suit, the injunction, first ruling, and appellate ruling were 7, 239, and 478 days respectively. In $80.6 \%$ of the 98 suits in which the specific medicines could be identified, at least one drug did not belong to any publicly funded list of medicines. This could indicate that lawsuits demanding "essential" drugs were motivated not only by problems in procurement, distribution, and dispensing of medicines but also by non-inclusion of medicines on official lists. Most of the medicines demanded through lawsuits were for conditions involving the cardiovascular and nervous systems.
\end{abstract}

Essential Drugs; Right to Health; Judicial Decisions; National Drug Policy; Single Health System
As leis e recomendações internacionais reconhecem o direito à saúde como um direito humano, estando ele incluso nas leis constitucionais de alguns países. O acesso aos medicamentos essenciais é considerado por alguns autores como integrante deste direito 1,2,3.

No Brasil, o direito à saúde está disposto nos artigos 6ọ, 196o a 200o da Constituição Federal brasileira, como um direito social, a ser garantido por meio de políticas, que, dentre outras tarefas, devem promover e garantir o acesso universal e igualitário às ações e serviços para promoção, proteção e recuperação da saúde dos cidadãos. A Lei Orgânica da Saúde (Lei Federal $n^{\circ}$. 8.080/90) ${ }^{4}$, em seus artigos 6o e 7o, regulamenta o sistema público de saúde, estabelecido constitucionalmente, e atribuiu ao Sistema Único de Saúde (SUS) a execução de ações de assistência terapêutica integral, inclusive farmacêutica. Desta forma, o reconhecimento da saúde como um direito possui duas importantes repercussões práticas: a responsabilidade ética e legal do poder público de formular e implementar ações que assegurem o acesso da população aos serviços de atenção à saúde; e a possibilidade do cidadão reivindicar judicialmente, de forma individual ou coletiva, o cumprimento desta obrigação estatal. Os chefes dos executivos da saúde têm formulado e implementado políticas e ações de saúde, inclusive de assistência farmacêutica, por meio 
de um amplo conjunto de instruções e normas administrativas sobre os mais variados aspectos da seleção, programação, aquisição, distribuição e dispensação de medicamentos. Essas normas são em sua maioria, de origem federal 5 .

A Política Nacional de Medicamentos (PNM) objetiva garantir o acesso da população aos medicamentos considerados essenciais 6 e remete a duas reflexões. A primeira é sobre o conceito de essencialidade que constitui pedra angular da PNM. Neste sentido, o desenvolvimento científico e tecnológico, a produção de medicamentos, a verificação de sua qualidade, a regulamentação sanitária, a reorientação da assistência farmacêutica e o desenvolvimento e capacitação de recursos humanos devem levar em conta a almejada melhoria do acesso aos medicamentos, em especial, aos essenciais 7,8,9,10.

A segunda reflexão diz respeito à recomendação da Organização Mundial da Saúde (OMS) 11 em relação à seleção de medicamentos, estabelecendo que cada país elabore um rol de medicamentos essenciais. O processo de adoção e revisão de uma lista de medicamentos essenciais traz implícito que a seleção deve seguir critérios que contemplem as necessidades terapêuticas de determinada população, proporcionando assim, base para priorização de necessidades, redundando na ampliação do acesso. Igualmente, a seleção de medicamentos essenciais propicia a melhoria da qualidade da assistência, pois, ao estabelecer como critérios de seleção a eficácia e a segurança dos fármacos, busca limitar o uso de medicamentos de eficácia não comprovada, que apresentam maiores riscos do que benefícios na sua utilização ou a duplicidade de fármacos para a mesma indicação clínica.

No Brasil, a Relação Nacional de Medicamentos Essenciais (RENAME) tem tido atualização periódica desde o ano de 2000, como recomendado pela OMS. É importante destacar que a definição de medicamentos essenciais não está relacionada estritamente ao custo financeiro do medicamento, sendo os de alto custo também incluídos nestas listas. Além da seleção dos medicamentos essenciais, o sistema de saúde público brasileiro possui, em suas três esferas de governo, outros elencos de medicamentos selecionados, tais como os de dispensação excepcional e os programas de distribuição de medicamentos para doenças específicas, como tuberculose, malária, diabetes e outras, de forma a atender diferentes demandas de saúde.

Apesar dos avanços nas políticas e ações públicas de assistência farmacêutica, no Brasil, diversos estudos 7,8,12,13 identificam dificuldades de acesso da população aos medicamentos necessários, o que implica prejuízo à assistência integral à saúde. Neste contexto, é compreensível que o cidadão busque um tipo de alternativa mais rápida e efetiva de acesso aos medicamentos.

No Estado do Rio de Janeiro e no Brasil, o Poder Judiciário tem se revelado como uma alternativa de obtenção deste fornecimento. Deste modo, estabelece-se uma tensão entre o Poder Judiciário, cuja atribuição constitucional é fazer cumprir as leis que garantem o direito dos cidadãos aos medicamentos, e o Poder Executivo que tem como atribuições estabelecer e executar políticas que dêem cumprimento às leis produzidas pelo Poder Legislativo, de forma que permita à sociedade o acesso efetivo e equânime à assistência farmacêutica. O fenômeno, que tem sido chamado de "judicialização da saúde", expressa a problemática apontada, e os estudos sobre o tema revelam a crescente demanda judicial, individual e coletiva, por bens e serviços de saúde, principalmente medicamentos, presentes ou não em listas oficiais de fornecimento, para diversos tipos de indicações terapêuticas.

Conhecer a demanda individual e local por medicamentos, bem como os aspectos do rito processual que viabiliza este acesso "alternativo" ao cidadão, pode auxiliar os gestores do setor saúde e do sistema de justiça no desenho de estratégias que melhorem o acesso da população aos medicamentos. Sob esta orientação, esta pesquisa objetivou analisar as ações individuais de fornecimento de medicamentos, considerados essenciais pelo Tribunal de Justiça do Estado do Rio de Janeiro nas decisões judiciais de 2a instância (acórdãos) proferidas no ano de 2006.

\section{Método}

Foi realizado um estudo descritivo retrospectivo. A unidade de análise foi o processo judicial individual movido por cidadão contra o ente estatal (estado ou municípios) no Tribunal de Justiça do Estado do Rio de Janeiro (TJ/RJ), que tinha como objeto o fornecimento de medicamento. Compuseram o estudo, os processos julgados na 2a instância no ano de 2006, em cujas ementas das decisões constavam os termos "medicamento" e "essencial". Os dados relativos às ações judiciais selecionadas foram obtidos por consulta às ementas disponibilizadas no sítio do TJ/RJ na Internet (http://www.tjrj.jus.br).

As informações foram coletadas por meio de instrumento semi-estruturado, abordando os elementos processuais e os elementos médicocientíficos e sanitários das demandas. As variáveis de interesse selecionadas foram: (a) elementos processuais: a comarca de origem da ação, relacionada à residência do autor; o réu; o número 
de autores da ação; o patrocinador (Defensoria Pública ou advogado particular); a existência de despacho judicial exigindo cumprimento, pelo autor, de algum requisito, anterior à ordem liminar; tipo de decisão judicial (deferimento, deferimento parcial ou indeferimento do pedido do autor); existência de recurso judicial; datas (início da ação, decisão de antecipação da tutela (liminar), sentença na 1a instância; e entrada e decisão na 2a instância) e (b) elementos médicocientíficos e sanitários: medicamentos pleiteados; registro do medicamento na Agência Nacional de Vigilância Sanitária (ANVISA); presença do medicamento nas listas oficiais de fornecimento público; condição patológica do autor.

Os medicamentos solicitados nas ações foram classificados de acordo com a recomendação da OMS, pela Anatomical Therapeutic Chemical Classification (ATC) 14 e as doenças identificadas foram classificadas pela décima revisão da Classificação Internacional de Doenças (CID-10) 15. Foi considerado como diagnóstico principal a primeira doença mencionada, ou quando assim estava especificada no processo; e como diagnóstico(s) secundário(s) a(s) subseqüente(s). A presença do medicamento (na concentração e forma farmacêutica solicitada) nas listas oficiais de fornecimento gratuito de medicamentos foi verificada por meio da consulta à RENAME 2002 (vigente na ocasião). A verificação do registro do medicamento na ANVISA teve como fonte o respectivo sítio da agência reguladora na Internet (http://www.anvisa.gov.br).

Foi utilizado o aplicativo Epidata Entry versão 3.1 (Epidata Assoc., Odense, Dinamarca) para o preenchimento do instrumento. A entrada dos dados foi realizada com dupla digitação por farmacêuticos familiarizados com a sistemática dos processos e com a linguagem jurídica. Finalizadas a digitação e as eventuais correções, os dados foram analisados descritivamente no aplicativo Epidata Analysis versão 2.0.

Este trabalho é resultado do projeto de pesquisa intitulado A Judicialização no Acesso a Medicamentos no Estado do Rio de Janeiro: Um Olhar Sobre o Essencial, que foi aprovado pelo Comitê de Ética em Pesquisa da Escola Nacional de Saúde Pública Sergio Arouca, Fundação Oswaldo Cruz (protocolo 93/07) e financiado pela Fundação de Apoio à Pesquisa no Estado do Rio de Janeiro (FAPERJ).

\section{Resultados}

\section{Descrição dos elementos processuais}

Foram encontrados 185 processos judiciais, julgados no ano de 2006, com os descritores "medicamento" e "essencial", no sistema de informação da 2a instância do TJ/RJ. A Comarca da Capital contou com o maior número de processos, $36,6 \%$, seguida pela de Teresópolis com 19,8\% do total. As Comarcas do Rio de Janeiro, Teresópolis e Volta Redonda foram responsáveis por 70,9\% destas ações.

Em relação ao segundo elemento processual pesquisado, o Estado do Rio de Janeiro foi o que mais freqüentemente figurou como réu $(40,9 \%)$, seguido pelos municípios do Rio de Janeiro (30,9\%), de Teresópolis (18,2\%) e de Volta Redonda (13,8\%) (Tabela 1). Em 68 ações $(36,8 \%)$, estado e algum município figuravam como réus. Isto indica que os autores entenderam que ambos os entes federativos são os responsáveis pelo fornecimento dos medicamentos pleiteados. A maior

\section{Tabela 1}

Distribuição das ações individuais de medicamentos, segundo réu: ações de 2a instância. Estado do Rio de Janeiro, Brasil, 2006 *

\begin{tabular}{|c|c|c|}
\hline Réu & $\mathbf{n}$ & $\%$ \\
\hline Estado do Rio de Janeiro & 74 & 40,9 \\
\hline Município do Rio de Janeiro & 56 & 30,9 \\
\hline Teresópolis & 33 & 18,2 \\
\hline Volta Redonda & 25 & 13,8 \\
\hline Barra do Piraí & 15 & 8,3 \\
\hline Barra Mansa & 15 & 8,3 \\
\hline São Gonçalo & 7 & 3,9 \\
\hline Itaperuna & 5 & 2,8 \\
\hline Nova Iguaçu & 4 & 2,2 \\
\hline Duque de Caxias & 3 & 1,7 \\
\hline Nova Friburgo & 2 & 1,1 \\
\hline Três Rios & 2 & 1,1 \\
\hline Valença & 2 & 1,1 \\
\hline Angra dos Reis & 1 & 0,6 \\
\hline Nilópolis & 1 & 0,6 \\
\hline Niterói & 1 & 0,6 \\
\hline Pinheiral & 1 & 0,6 \\
\hline Resende & 1 & 0,6 \\
\hline São Pedro da Aldeia & 1 & 0,6 \\
\hline
\end{tabular}

Fonte: Tribunal de Justiça do Estado do Rio de Janeiro

(http://www.tjrj.jus.br), elaboração própria.

* Pode haver mais de um réu numa mesma ação. 
parte delas (55 ações) tinha como réus o estado e o Município do Rio de Janeiro.

A maioria das ações (98,9\%) tinha um único autor e $83 \%$ dos autores foram representados pela Defensoria Pública.

Em apenas 9,6\% das ações houve algum tipo de exigência judicial anterior à concessão da ordem liminar, para a entrega imediata pelo Poder Público do medicamento, sendo a mais freqüente a comprovação de hipossuficiência financeira. As demais exigências referiam-se à regularização de documentos civis e não médicos.

Em $100 \%$ dos processos judiciais selecionados, o juiz concedeu liminarmente o fornecimento dos medicamentos pleiteados, admitindo a urgência dos pedidos dos autores em face do alegado dano irreversível à sua saúde, e o dever do Estado de fornecimento do medicamento. Neste sentido, o juiz deferiu o pedido liminar sem ouvir o réu, ou requerer provas adicionais às já apresentadas pelo autor para a concessão da medida excepcional de antecipação da tutela judicial (liminar), conforme autoriza a lei processual. Porém, em três sentenças finais foram negados pedidos referentes a fraldas descartáveis, a suplemento alimentar e a medicamento prescrito por médico não vinculado ao SUS.

Os réus recorreram em $13,4 \%$ das decisões liminares, em 71,4\% das sentenças de 1ạ instância. Em 24,5\% das decisões da segunda instância, identificou-se recurso especial ou extraordinário para os Tribunais Superiores.

A análise dos tempos medianos entre a entrada do processo na primeira instância e a decisão liminar foi de sete dias; entre a decisão liminar e a sentença o tempo foi de 218 dias; e entre a entrada do processo na 1a instância e a sentença 239 dias. O tempo mediano entre a entrada na primeira instância e a decisão terminativa na segunda instância foi de 478 dias. A mediana dos tempos entre a entrada na segunda instância e a decisão final foi de 49 dias (Tabela 2).
Descrição dos elementos médico-científicos e sanitários

Foi possível a identificação dos medicamentos pleiteados em apenas 98 ações (53\%). Verificouse uma média de 3,25 medicamentos solicitados por processo judicial. Foram encontradas 321 solicitações de medicamento e 307 puderam ser classificados até o quinto nível da ATC, totalizando 149 diferentes fármacos. Os grupos anatômicos mais freqüentes foram: o sistema cardiovascular $(31,6 \%)$ e o sistema nervoso central $(23,1 \%)$ (Tabela 3). Os fármacos solicitados com maior freqüência foram furosemida, ácido acetilsalicílico e digoxina (2,9\% cada), seguidos do enalapril (2,6\%), propatilnitrato (2\%), clonazepam ( $2 \%)$ e captopril $(1,6 \%)$.

A existência de registro na ANVISA pôde ser verificada para 320 dos medicamentos solicitados (uma solicitação se referia à fórmula magistral). Destes, apenas três $(0,9 \%)$ não possuíam registro na ANVISA, sendo que para dois havia registro do princípio ativo, porém não na concentração prescrita.

Dos 321 medicamentos identificados nas ações judiciais, 316 foram classificados segundo presença na RENAME, já que para cinco faltaram informações indispensáveis como dose e forma farmacêutica. Do total identificado, 35,8\% estavam presentes na RENAME 2002 e 48,1\% estavam presentes em alguma lista oficial (RENAME e/ou demais listas oficiais). Das 98 ações judiciais em que os medicamentos requeridos puderam ser identificados, $80,6 \%$ solicitava pelo menos um medicamento (média de 1,7 por ação) não pertencente ao elenco da RENAME nem das outras listas oficiais de medicamentos.

Dos medicamentos não presentes na RENAME, o propatilnitrato foi solicitado com maior freqüência $(3,8 \%)$, seguido do mononitrato de isossorbida, do anlodipino, do alendronato de sódio, do bromazepam, da memantina e da

Resumo dos tempos (em dias) dos processos originários na 1ạ instância. Estado do Rio de Janeiro, Brasil, 2006.

\begin{tabular}{|c|c|c|c|c|c|c|}
\hline & Total de ações & Mínimo & Máximo & Média & Mediana & Desvio-padrão \\
\hline Tempo entre a entrada e a decisão liminar & 157 & 0 & 276 & 19 & 7 & 41 \\
\hline Tempo entre a entrada e a sentença na 1ạ instância & 160 & 28 & 1362 & 266 & 239 & 176 \\
\hline Tempo entre a entrada na 2ạ instância e a decisão na 2ạ instância & 155 & 1 & 295 & 67 & 49 & 65 \\
\hline Tempo entre a decisão liminar e a sentença & 155 & 22 & 757 & 239 & 218 & 150 \\
\hline Tempo entre a entrada na 1ạ instância e a decisão na 2a instância & 150 & 129 & 1534 & 503 & 478 & 233 \\
\hline
\end{tabular}

Fonte: Tribunal de Justiça do Estado do Rio de Janeiro (http://www.tjrj.jus.br), elaboração própria. 
Distribuição dos medicamentos solicitados, segundo classificação pelo primeiro nível do Anatomical Therapeutic Chemical Classification (ATC) 14: ações de 2ạ instância. Estado do Rio de Janeiro, Brasil, 2006.

\begin{tabular}{llc}
\hline Primeiro nível da ATC & $\mathbf{n}$ & \% \\
\hline C. Sistema cardiovascular & 97 & 31,6 \\
N. Sistema nervoso central & 71 & 23,1 \\
A. Aparelho digestivo e metabolismo & 42 & 13,7 \\
R. Sistema respiratório & 25 & 8,1 \\
B. Sangue e órgãos hematopoiéticos & 19 & 6,2 \\
L. Antineoplásicos e agentes moduladores do sistema imunológico & 16 & 5,2 \\
G. Sistema genito urinário e hormônios sexuais & 11 & 3,6 \\
M. Sistema músculo esquelético & 11 & 3,6 \\
H. Hormônios de uso sistêmico, excluindo hormônios sexuais & 8 & 2,6 \\
J. Antiinfecciosos gerais para uso sistêmico & 4 & 1,3 \\
S. Órgãos dos sentidos & 3 & 1,0 \\
Total & 307 & 100,0
\end{tabular}

Fonte: Tribunal de Justiça do Estado do Rio de Janeiro (http://www.tjrj.jus.br), elaboração própria.

combinação salmeterol/fluticasona (2,5\% cada). É importante ressaltar que alguns medicamentos como anlodipino, atenolol, glicazida, alendronato de sódio, carvedilol e enalapril, não pertenciam à RENAME 2002 - relação vigente na data da decisão judicial - mas foram incluídos em revisões posteriores da RENAME (2006 e 2008).

Das 185 ações, em 133 (71,2\%) foi possível identificar pelo menos um diagnóstico, perfazendo um total de 239 morbidades. Das mesmas, apenas 14 (4,8\%) continha o CID-10 classificada pelo médico. Os diagnósticos principais mais freqüentes, segundo os agrupamentos da CID-10, foram os de doenças hipertensivas $(12,8 \%)$, diabetes mellitus $(11,3 \%)$, outras doenças degenerativas do sistema nervoso, doenças crônicas das vias aéreas inferiores e insuficiência renal (5,3\% cada). A seleção de todas as morbidades mencionadas, incluindo os diagnósticos principais e secundários, apresentou resultado semelhante: as morbidades mais mencionadas, por agrupamento da CID-10, foram doenças hipertensivas $(11,3 \%)$, diabetes mellitus $(9,2 \%)$, doenças crônicas das vias aéreas inferiores e transtornos da densidade e da estrutura óssea (3,8\% cada) (Tabela 4$)$

\section{Discussão}

O estudo mostrou as características processuais e médico-sanitárias de demandas judiciais no Estado do Rio de Janeiro, no ano de 2006, de medicamentos considerados essenciais nas ementas das decisões da segunda instância do Tribunal de Justiça. No momento da pesquisa foi constatada a inexistência de lista estadual de medicamentos essenciais. Nesta análise, portanto, a baliza para verificação da essencialidade foi a RENAME, uma vez que a lista nacional se pauta em critérios epidemiológicos, de eficácia e de segurança, critérios definidores de essencialidade para o setor saúde.

O processo de informatização dos tribunais permite a publicidade de suas decisões, viabilizando o conhecimento por parte dos cidadãos, o monitoramento dos atos judiciais como um mecanismo de controle social, e também a realização de estudos, como este. Porém, uma de suas limitações é a impossibilidade de acesso, via Internet, de todas as informações necessárias ao estudo, como "medicamentos pleiteados" e "condição patológica" do autor, que foram identificados, em apenas 53\% e $71 \%$ das ações, respectivamente.

A grande concentração da demanda judicial na capital (36,6\%), onde há um maior contingente populacional, pode estar relacionada com a existência de uma maior rede de serviços de saúde e jurídicos. A maior demanda judicial em determinados municípios merece análise mais aprofundada, não tendo sido possível, nos limites desta pesquisa, identificar as razões com relação aos municípios de Teresópolis, Volta Redonda e Barra Mansa, e a ausência quase completa de demanda nos outros municípios.

A identificação de $36,8 \%$ de ações judiciais propostas com mais de um ente federativo como 
Distribuição dos diagnósticos, principal e secundários, por agrupamento da décima revisão da Classificação Internacional de Doenças (CID-10) 15: ações de 2ạ instância. Estado do Rio de Janeiro, Brasil, 2006.

\begin{tabular}{|c|c|c|c|}
\hline Agrupamento da CID-10 & Morbidade & $\mathrm{n}$ & $\%$ \\
\hline$|10-| 15$ & Doenças hipertensivas & 27 & 11,3 \\
\hline E10-E14 & Diabetes mellitus & 22 & 9,2 \\
\hline J40-J47 & Doenças crônicas das vias aéreas inferiores & 9 & 3,8 \\
\hline M80-M85 & Transtornos da densidade e da estrutura óssea & 9 & 3,8 \\
\hline F30-F39 & Transtornos do humor (afetivos) & 8 & 3,3 \\
\hline G40-G47 & Transtornos episódicos e paroxísticos (sistema nervoso central) & 8 & 3,3 \\
\hline N17-N19 & Insuficiência renal & 8 & 3,3 \\
\hline G30-G32 & Outras doenças degenerativas do sistema nervoso & 7 & 2,9 \\
\hline G80-G83 & Paralisia cerebral e outras síndromes paralíticas & 7 & 2,9 \\
\hline $120-125$ & Doenças isquêmicas do coração & 7 & 2,9 \\
\hline $130-152$ & Outras formas de doença do coração & 7 & 2,9 \\
\hline G20-G26 & Doenças extrapiramidais e transtornos dos movimentos & 6 & 2,5 \\
\hline Q00-Q07 & Malformações congênitas do sistema nervoso & 6 & 2,5 \\
\hline K50-K52 & Enterites e colites não-infecciosas & 5 & 2,1 \\
\hline- & Outras morbidades & 103 & 43,3 \\
\hline Total & & 239 & 100,0 \\
\hline
\end{tabular}

Fonte: Tribunal de Justiça do Estado do Rio de Janeiro (http://www.tjrj.jus.br), elaboração própria.

réu (estado e município), indica a adoção pelo reivindicante da tese da responsabilidade solidária de municípios e estado na assistência farmacêutica. O TJ/RJ admite em sua jurisprudência esta tese, que significa afirmar que é de competência comum dos entes federativos o fornecimento de medicamentos, e que as pactuações entre eles, por exemplo, relacionadas ao financiamento e à distribuição dos medicamentos, são válidas e devem prevalecer entre os co-responsáveis pela prestação, mas estas pactuações não podem servir como entrave para o acesso da população ao seu direito à assistência farmacêutica.

A tese da solidariedade dos entes federativos no cumprimento das prestações de saúde é um tipo de interpretação legal que decorre das competências e atribuições comuns dos entes federativos relativas ao cuidado da saúde estabelecidas constitucionalmente (Art. 23o da Constituição Federal) e na Lei Federal $n^{o}$. 8.080/90 (Art. 15으) 4. A figura jurídica da responsabilidade solidária representa um instrumento legal que visa ampliar a garantia do credor de uma prestação em relação àqueles que são co-obrigados a prestá-la.
A repercussão prática, no âmbito das ações judiciais de fornecimento de medicamento, é que esta tese favorece o acesso mais rápido e efetivo ao insumo pleiteado, sem impedir que o ente federativo condenado realize a cobrança das prestações pagas ao ente responsável, conforme pactuações firmadas entre eles, por via judicial ou administrativa.

Importa destacar que não há lei federal que defina a qual ente federativo caberá o fornecimento de determinado rol de medicamentos. Esta divisão de responsabilidade é feita por negociação entre os entes federativos e expressa por meio de normas administrativas (Portarias, Resoluções, Normas Técnicas, Protocolos Terapêuticos), geralmente resultado de pactuações realizadas entre as instâncias do SUS, tais como a Comissão Intergestores Tripartite (composta por representação de gestores da esfera federal, estadual e municipal) e a Comissão Intergestores Bipartite (composta por representação de gestores da esfera estadual e municipal). Estas normas nem sempre são claras em relação a que medicamentos são de responsabilidade de cada nível 
de gestão. Além disso, a regulamentação da assistência farmacêutica é bastante fragmentada, e sofre muitas alterações, sendo de difícil identificação e compreensão, como expressa o estudo de Pontes Júnior 9 . Portanto, o cidadão ao ajuizar ação em face de mais de um réu e o TJ/RJ adotar a tese da solidariedade dos entes federativos pode indicar, respectivamente, uma estratégia de litigância e uma política judicial, que visam aumentar a garantia do cidadão ao recebimento rápido do medicamento urgente pleiteado.

O predomínio de ações judiciais patrocinadas pela Defensoria Pública (83\%) foi um aspecto revelador do perfil do demandante no Estado do Rio de Janeiro e da atuação desta instituição no acesso à justiça e ao sistema público de saúde. O predomínio da atuação da Defensoria Pública neste âmbito também foi observado por Messeder et al. 16, no Estado do Rio de Janeiro, e por Romero 17, no Distrito Federal. Estudos realizados em outros estados - Vieira \& Zucchi 18, no Município de São Paulo; Marques \& Dallari 19, no Estado de São Paulo e Pereira et al. 20, no Estado de Santa Catarina - referiram ser a maior parte das ações conduzidas por advogados particulares. Há dois aspectos que devem ser aprofundados para entendermos este dado. Um é em relação à consolidação da Defensoria Pública nos estados. O outro aspecto relaciona-se aos critérios adotados para o reconhecimento da hipossuficiência dos autores na demanda judicial.

A estruturação da Defensoria Pública é considerada constitucionalmente (Art. 134o da Constituição Federal) uma instituição essencial à realização da Justiça e, neste sentido, a atuação desta instituição constitui uma importante variável de análise para a compreensão da demanda judicial em saúde. A criação e organização das Defensorias Públicas são de competência estadual. Mas, alguns estados brasileiros ainda não possuem Defensorias Públicas, e em outros sua instalação é muito recente. Na ausência de Defensorias Públicas existe a prestação de advocacia para os hipossuficientes feita por advogados privados, pagos não pelo autor da ação, mas pelo poder público, por meio de convênios com instituições que prestam este serviço, as Defensorias Dativas 21. A Defensoria Pública do Estado do Rio de Janeiro é uma instituição bastante antiga. Está expressa na Constituição Estadual do ano de 1975, antes mesmo da garantia na Constituição Federal, mas sua criação data do ano de 195421. A Defensoria Pública no Estado de São Paulo, por exemplo, somente foi instituída em 2006; e o Estado de Santa Catarina até os dias atuais não possui Defensoria Pública, e na última pesquisa identificada, não constam convênios com instituições para Advocacia Dativa 21. Neste sentido, é importante aprofundar os dados de alguns estudos realizados em estados que não possuíam Defensorias Públicas. Importante ainda ressaltar que o autor, mesmo representado por advogados particulares, pode ser beneficiário da gratuidade da justiça, que permite a isenção de custas e outras despesas processuais para os necessitados e a atuação de advogados dativos.

No Estado do Rio de Janeiro e no Distrito Federal, locais onde predomina a representação da Defensoria Pública, a comprovação da hipossuficiência da pessoa é requisito para ser atendido, sendo esta condição avaliada individualmente por meio de análise documental e declaração pessoal do requerente, quanto às suas necessidades. Em outros estados, como São Paulo, Minas Gerais e Rio Grande do Sul, um dos critérios para o acesso à Defensoria Pública é uma renda familiar máxima de três salários mínimos 21 . No Estado do Rio de Janeiro, o reconhecimento judicial da hipossuficiência do reivindicante é uma característica majoritária da demanda de medicamentos, não sendo possível afirmar que ela viole a equidade do SUS, no sentido de estar atendendo a uma classe econômica mais favorecida em detrimento dos menos favorecidos, como outros estudos apontam 2,18 .

A antecipação de tutela judicial - comumente chamada de "decisão liminar” - é também variável processual de interesse na análise das demandas judiciais de medicamentos. Nos casos analisados neste estudo o pedido dos autores foi integralmente concedido. A antecipação de tutela é um tipo de decisão judicial, na qual o juiz deve analisar alguns requisitos essenciais do pedido do autor: (1) se há verossimilhança dos fatos alegados, isto significa considerar se a prova apresentada pelo reivindicante possui fortes indícios de ser verdadeira; (2) se há "perigo da demora" da prestação jurisdicional, ou seja, se o juiz autorizar o fornecimento após a instrução de todo procedimento judicial (na sentença) esta demora pode resultar em violação irreparável do direito autor, p.ex., agravamento de seu estado de saúde; (3) se há "fumaça do bom direito", isto é, se o pedido do autor está amparado por lei. Após a concessão da "liminar", o procedimento continuará, e caso o juiz verifique, ao final, que a prestação solicitada não era devida, o autor deve indenizar o réu. O réu, por sua vez, pode se insurgir contra a antecipação de tutela deferida, apresentando provas contrárias à pretensão do requerente. A tutela antecipada, então, poderá ser revista pelo próprio juiz que a concedeu, ou por instâncias colegiadas superiores.

A concessão de todas as liminares solicitadas e o baixo número de exigências iniciais determinadas pelo julgador na análise do pedido de 
tutela antecipada, sendo estas, sobretudo restritas à comprovação de hipossuficiência, parece reforçar o que outros estudos têm evidenciado: que o principal critério judicial para a concessão da tutela é a constatação da necessidade do autor de acesso urgente a determinado medicamento ou procedimento, tendo como respaldo os documentos fornecidos pelo demandante, em geral, os receituários médicos 17,18,19,22.

Vale lembrar que diversos fatores podem influenciar negativamente a qualidade da prescrição médica e que, portanto, prescrições inadequadas não são incomuns ${ }^{23}$. A publicidade de medicamentos, por exemplo, exerce forte influência sobre o padrão de prescrição dos médicos na ausência de ferramentas eficazes de controle sobre a veracidade das informações 24 .

A quase totalidade de decisões finais concedendo integralmente os pedidos dos autores e o baixíssimo percentual de recursos refletem as fragilidades da defesa dos Poderes Executivos, réus nas ações judiciais, como do Poder Judiciário para lidar com a questão. Essas fragilidades podem resultar em possíveis danos ao paciente, no caso de uma prescrição inadequada. O estudo mostrou que o tempo entre a entrada na primeira instância e a decisão terminativa na segunda instância, que analisa o recurso do réu é de 478 dias, o que torna possíveis erros de prescrição em efetiva falha de segurança da terapêutica.

Quanto à descrição dos medicamentos solicitados nas ações, os resultados encontram semelhanças com o estudo realizado por Messeder et al. 16, que identificou os fármacos furosemida e captopril como os mais freqüentemente solicitados. Ressalta-se que estes medicamentos são itens de atenção básica, de competência de fornecimento pelos municípios. Não houve, nas ações analisadas no estudo, significativa solicitação de medicamentos não registrados no país $(0,9 \%)$.

Os estudos realizados até o momento identificaram que, considerando o total de medicamentos solicitados nas ações, parte considerável deles estava presente em listas oficiais, de forma semelhante ao desta pesquisa, apontando para a existência de problemas relacionados à aquisição, distribuição e dispensação de medicamentos pelo setor público 2,16,17,18,19,25. Aprofundando os resultados, de forma inovadora, foi realizada a análise individualizada dos pedidos de medicamentos demandados em cada processo vis-a-vis sua presença nas listas oficiais. Em 98 processos o medicamento pode ser identificado e em $80,6 \%$ havia solicitação de ao menos um medicamento não pertencente aos elencos oficiais. Isto sugere que, além dos problemas de aquisição, distribuição e dispensação de itens listados, os medica- mentos não inclusos em listas oficiais são uma forte motivação da demanda judicial.

Ressalta-se, ainda, que a posterior inclusão de medicamentos na RENAME (anlodipino, atenolol, glicazida, alendronato de sódio, carvedilol e enalapril) pode indicar que a judicialização se deu, nestes casos, por atraso na incorporação destes medicamentos.

O predomínio de doenças crônicas entre as condições patológicas dos autores das ações pode, em parte, ser explicado pela necessidade de medicamentos de uso continuado, muitas vezes de alto custo. Nos estudos realizados por Messeder et al. 16 e por Borges 25, também no Estado do Rio de Janeiro, doenças crônicas, como as do sistema cardiovascular, do sistema nervoso e do trato alimentar ou metabolismo também figuraram entre as mais freqüentes nos pleitos. Resultados semelhantes foram encontrados em estudos de outros Estados da Federação 2,17,18,20.

\section{Considerações finais}

O estudo ora realizado justificou-se frente à importância que a demanda judicial de acesso aos medicamentos vem assumindo para os gestores da saúde e os desafios para atuação cooperativa e efetiva entre o setor saúde e o sistema de justiça em prol da saúde da população. A "essencialidade" do medicamento na perspectiva do setor saúde, corresponde à definição expressa na PNM de garantir o acesso aos medicamentos considerados essenciais, e no papel de destaque conferido à RENAME. Nas decisões judiciais analisadas, a "essencialidade" está relacionada à necessidade terapêutica do reivindicante ao medicamento pleiteado.

A análise das ações judiciais individuais de acesso a medicamentos no Rio de Janeiro, considerados essenciais pelo Poder Judiciário na segunda instância, trouxe à luz alguns avanços para a compreensão do perfil desta demanda no TJ/RJ, na perspectiva do conceito de essencialidade. Avança também, do ponto de vista operacional, para pensar ações na melhoria da prestação de saúde e jurisdicional, e na articulação destes setores, identificando as principais características desta demanda judicial no estado.

Este estudo traz, ainda, importante contribuição adicional aos já realizados por sugerir que, nesta amostra, a busca do medicamento por meio judicial envolveu, de modo consistente, aqueles não inclusos nas listas oficiais do SUS, fato que merece estudos mais aprofundados sobre as motivações dos demandantes.

Outro significativo avanço é o fato de que esta análise pôde se debruçar sobre o processo 
judicial em quase toda a sua trajetória, desde a entrada na 1a instância até a decisão na 2ạ instância. A análise do tempo transcorrido nas diferentes etapas processuais constitui-se em um avanço teórico e prático, uma vez que não foi identificado estudo anterior desta natureza em ações correlacionadas ao fornecimento de medicamentos. A definição do tempo de tramitação entre as diferentes etapas do processo judicial permitiu identificar a rápida tramitação quando visto sob a ótica do setor de justiça.

O deferimento absoluto dos pedidos de tutela antecipada de medicamentos, aliado ao tempo mediano entre decisão liminar de antecipação de tutela e o julgamento do mérito da ação (214 dias) e ao baixo percentual de recursos da liminar interpostos pelos réus, indicam que a maioria das decisões liminares continua sendo cumprida por meses sem que haja uma correta avaliação ou contestação do Executivo da Saúde da adequação do pleito dos cidadãos. O juiz inicialmente concede a tutela em caráter de urgência com poucos subsídios clínicos; o Executivo da Saúde opta por não recorrer da decisão liminar; e o cidadão, autor da ação e enfermo, permanece por longo tempo fazendo uso de um medicamento que, de fato, pode não estar resguardando sua saúde. Do ponto de vista médico-sanitário e considerando a possibilidade de prescrição inadequada, pode representar para o paciente um longo período de uso irracional de medicamento, que pode comprometer ainda mais sua já combalida saúde.
As normas legais relativas ao acesso à prestação jurisdicional e de saúde são diferenciadas, sendo o acesso ao SUS gratuito, por princípio, para todo cidadão, já na Defensoria Pública e no Poder Judiciário há uma avaliação da hipossuficiência do autor para conferir-lhe a gratuidade. Neste sentido, pode-se inferir que o reivindicante beneficiado com a gratuidade de justiça e representado pela Defensoria Pública também não possui condições para arcar com os custos de seu tratamento, considerando que as despesas judiciais em geral são pontuais e bem menores do que as de despesas com alguns tratamentos de saúde. Porém, a característica da hipossuficiência dos demandantes judiciais merece ser problematizada e aprofundada, sobretudo no que se refere ao estabelecimento de critérios para o alcance da equidade no acesso ao SUS e ao sistema de justiça.

$\mathrm{O}$ adequado enfrentamento, a bem do paciente/demandante, exige maior interação e ações mais efetivas dos setores de saúde e de justiça, que possam superar as limitações de ambos os sistemas e respondam de forma adequada e específica às demandas individuais judiciais na saúde que visam o fim último de preservar ou recuperar a saúde da pessoa. Uma atualização periódica das listas oficiais ou a existência de caminhos administrativos no SUS, que avaliem a necessidade de medicamentos ainda não incorporados, podem garantir o acesso aos medicamentos essenciais, evitando as ações judiciais.

\section{Resumo}

O reconhecimento do direito à saúde possui duas repercussões práticas: a responsabilidade ética e legal do poder público em assegurar atenção integral à saúde da população, e a possibilidade de o cidadão reivindicar judicialmente o cumprimento desta obrigação estatal. Este estudo objetivou pesquisar as demandas de medicamentos considerados essenciais nas decisões da 2a instância do Tribunal de Justiça do Estado do Rio de Janeiro, Brasil, em 2006. Foram analisados 185 processos e em três casos o pedido foi negado. Os tempos medianos entre início da ação e decisão liminar, sentença na 1 a instância, e acórdão na 2 a instância, foram de 7, 239 e 478 dias, respectivamente. Em 98 processos o medicamento pode ser identificado e em $80,6 \%$ havia solicitação de ao menos um medicamento não pertencente aos elencos oficiais. Isto sugere que, além de problemas relacionados à aquisição, distribuição $e$ dispensação de itens listados, os não selecionados em listas oficiais foram também grande motivação da demanda. Os medicamentos mais solicitados foram os dos sistemas cardiovascular e nervoso central.

Medicamentos Essenciais; Direito à Saúde; Decisões Judiciais; Política Nacional de Medicamentos; Sistema Único de Saúde 


\section{Colaboradores}

V. L. E. Pepe contribuiu na concepção da idéia central do trabalho, análise dos dados e redação do texto. M. Ventura colaborou na concepção da idéia central do trabalho, coleta dos dados, análise dos dados e redação do texto. J. M. B. Sant'ana e T. A. Figueiredo participaram da coleta dos dados, construção do banco de dados, análise dos dados e redação do texto. V. R. Souza e L. Simas contribuíram na análise dos dados quantitativos e redação do texto. C. G. S. Osorio-de-Castro participou na redação e revisão do texto final.

\section{Referências}

1. Hogerzeil HV, Samson M, Casanovas JV, RahmaniOcora L. Is access to essential medicines as part of the fulfilment of the right to health enforceable through the courts? Lancet 2006; 368:305-11.

2. Chieffi AL, Barata RB. Judicialização da política pública de assistência farmacêutica e eqüidade. Cad Saúde Pública 2009; 25:1839-49.

3. Hogerzeil HV. Essential medicines and human rights: what can they learn from each other? Bull World Health Organ 2006; 84:371-5.

4. Brasil. Lei Federal ${ }^{\circ}$. 8.080, de 19 de setembro de 1990. Dispõe sobre as condições para a promoção, proteção e recuperação da saúde, a organização e o funcionamento dos serviços correspondentes e dá outras providências. Diário Oficial da União 1990; 19 set.

5. Pepe VLE, Ventura M. Assistência farmacêutica em foco no Estado do Rio de Janeiro: normas e documentos para ação [CD-ROM]. Rio de Janeiro: Escola Nacional de Saúde Pública, Fundação Oswaldo Cruz; 2009.

6. Brasil. Portaria $\mathrm{n}^{\circ}$. 3.916/MS/GM, de 30 de outubro de 1998. Aprova a Política Nacional de Medicamentos. Diário Oficial da União 1998; 10 nov.

\section{Agradecimentos}

À Fundação de Amparo à Pesquisa do Estado do Rio de Janeiro (FAPERJ), que financiou essa pesquisa por meio do projeto A Judicialização no Acesso a Medicamentos no Estado do Rio de Janeiro: Um Olhar Sobre o Essencial. Às pesquisadoras de campo, Letícia Figueira Freitas e Fernanda Affonso de Paula.
7. Ministério da Saúde/Organização Pan-Americana da Saúde/Organização Mundial da Saúde. Avaliação da assistência farmacêutica no Brasil: estrutura, processo e resultados. Brasília: Ministério da Saúde; 2005.

8. Guerra Jr. AA, Acúrcio FA, Gomes CAP, Miralles M, Girardi SN, Werneck GAF, et al. Disponibilidade de medicamentos essenciais em duas regiões de Minas Gerais, Brasil. Rev Panam Salud Pública 2004 15:168-75.

9. Pontes Junior DM. A seleção de medicamentos para o monitoramento da qualidade laboratorial no Brasil: articulação entre a Vigilância Sanitária e a Política Nacional de Medicamentos [Dissertação de Mestrado]. Rio de Janeiro: Escola Nacional de Saúde Pública, Fundação Oswaldo Cruz; 2007.

10. Pepe VLE, Osorio-de-Castro CGS, Luiza VLL. Relação Nacional de Medicamentos Essenciais: um instrumento da Política Nacional de Medicamentos na garantia do acesso. In: Buss PM, Carvalheiro JR, Casas CPR, organizadores. Medicamentos no Brasil: inovação e acesso. Rio de Janeiro: Editora Fiocruz; 2008. p. 319-34. 
11. Organización Mundial de la Salud. Perspectivas políticas sobre medicamentos de la OMS. 4. Selección de medicamentos esenciales. Geneva: Organización Mundial de la Salud; 2002.

12. Szwarcwald CL, Viacava F, Vasconcellos MTL, Leal MC, Azevedo LO, Queiroz RSB, et al. Pesquisa Mundial de Saúde 2003: o Brasil em números. RADIS Comunicação em Saúde 2004; 23:14-33.

13. Oliveira BB. Investigações de acesso a medicamentos em nível domiciliar: um estudo comparativo de iniciativas realizadas no Brasil entre 2002 e 2004 [Dissertação de Mestrado]. Rio de Janeiro: Escola Nacional de Saúde Pública, Fundação Oswaldo Cruz; 2007.

14. Capellà D. Descritive tools and analysis. In: Dukes MNG, editor. Drug utilization studies: methods and uses. Copenhagen: WHO Regional Publications; 1993. p. 55-78. (European Series, 45).

15. Organização Mundial da Saúde. Classificação estatística internacional de doenças e problemas relacionados à saúde, 10a revisão. v. 1. São Paulo: Centro Colaborador da OMS para a Classificação de Doenças em Português; 1995.

16. Messeder AM, Osorio-de-Castro CGS, Luiza VL. Mandados judiciais como ferramenta para garantia do acesso a medicamentos no setor público: a experiência do Estado do Rio de Janeiro, Brasil. Cad Saúde Pública 2005; 21:525-34.

17. Romero LC. Judicialização das políticas de assistência farmacêutica: o caso do Distrito Federal. Brasília: Consultoria Legislativa do Senado Federal; 2008. (Textos para Discussão, 41).
18. Vieira FS, Zucchi P. Distorções causadas pelas ações judiciais à política de medicamentos no Brasil. Rev Saúde Pública 2007; 41:214-22.

19. Marques SB, Dallari SG. Safeguarding of the social right to pharmaceutical assistance in the state of São Paulo, Brazil. Rev Saúde Pública 2007; 41:101-7.

20. Pereira JR, Santos RI, Nascimento Junior JM, Schenkel EP. Análise das demandas judiciais para o fornecimento de medicamentos pela Secretaria de Estado da Saúde de Santa Catarina nos anos de 2003 e 2004. Ciênc Saúde Coletiva; no prelo.

21. Ministério da Justiça. III diagnóstico da Defensoria Pública no Brasil. Brasília: Ministério da Justiça; 2009.

22. Sant'ana JMB. Essencialidade e assistência farmacêutica: um estudo exploratório das demandas judiciais individuais para acesso a medicamentos no Estado do Rio de Janeiro [Dissertação de Mestrado]. Rio de Janeiro: Escola Nacional de Saúde Pública, Fundação Oswaldo Cruz; 2009.

23. Brundtland GH. Global partnerships for health. WHO Drug Information 1999; 13:61-4.

24. Fagundes MJD, Soares MGA, Diniz NM, Pires JR, Garrafa V. Análise bioética da propaganda e publicidade de medicamentos. Ciênc Saúde Coletiva 2007; 12:221-9.

25. Borges DLC. Uma análise das ações judiciais para o fornecimento de medicamentos no âmbito do SUS: o caso do Estado do Rio de Janeiro no ano de 2005 [Dissertação de Mestrado]. Rio de Janeiro: Escola Nacional de Saúde Pública, Fundação Oswaldo Cruz; 2007.

Recebido em 10/Jul/2009

Versão final reapresentada em 17/Nov/2009

Aprovado em 07/Jan/2010 